\section{Abortion and breast cancer risk for Australian women}

To THE EDIToR: Recent comments in the media have renewed attention on the question of whether abortion is a risk factor for breast cancer, despite broad acceptance internationally in the scientific, medical and cancer advocacy communities that there is no association.

It was demonstrated in 2004 that abortion is not a risk factor for breast cancer, based on a combined analysis of prospective cohort study data from around the world. ${ }^{1}$ The authors showed empirically that retrospective casecontrol studies tend to give biased findings, most likely because women with breast cancer (cases) are more likely than controls to report previous abortions. Prospective cohort studies avoid this bias because information on abortions is collected before any of the women develop the disease. Indeed, a recent metaanalysis of findings from studies of Chinese women reported an apparent increased breast cancer risk associated with abortion based on case-control studies, but no evidence of association based on prospective cohort studies. ${ }^{2}$ Several other large prospective cohort studies have published consistently null results since $2004 .^{3,4}$

In 1990-1994, the Melbourne Collaborative Cohort Study ${ }^{5}$ recruited 24018 Victorian women aged 40-69 years who were free of breast cancer, and collected information on risk factors, including all pregnancies. The questionnaire did not differentiate between spontaneous and induced abortions. Using record linkage with the Victorian Cancer Registry, we determined that 1235 of these women were subsequently diagnosed with breast cancer, up to 30 December 2012. We assessed associations prospectively using
Cox regression, with age as the time variable, considered from recruitment to breast cancer diagnosis, and censored at death, permanent departure from Victoria or 30 December 2012. Analyses were adjusted for country, year of birth and socioeconomic status.

Ever having had an abortion in the first trimester was not associated with breast cancer risk (hazard ratio [HR], 1.06; 95\% CI, 0.94-1.20). The result was similar after additional adjustment for age at menarche, oral contraceptive use, number of full-term pregnancies, age at first birth and lifetime breastfeeding (HR, 1.07; 95\% CI, 0.94-1.22). The result was also similar for abortion before first full-term pregnancy (HR, 0.97; 95\% CI, 0.81-1.16) and for abortion in the first two trimesters (HR, $1.04 ; 95 \%$ CI, 0.92-1.17).

Consistent with international research findings, abortion is not associated with risk of breast cancer for Australian women.

Kelly-Anne Phillips Head, Breast and Ovarian Cancer Risk Management Clinic, and Honorary Professorial Fellow ${ }^{2}$

Fiona J Bruinsma Research Co-ordinator ${ }^{3}$

Roger L Milne Deputy Director, ${ }^{3}$ and Honorary Research Fellow ${ }^{2}$

1 Division of Cancer Medicine, Peter MacCallum Cancer Centre, Melbourne, VIC.

2 Centre for Epidemiology and Biostatistics, School of Population and Global Health, University of Melbourne Melbourne, VIC.

3 Cancer Epidemiology Centre, Cancer Council Victoria, Melbourne, VIC.

roger.milne@cancervic.org.au

Acknowledgements: Recruitment for the Melbourne Collaborative Cohort Study was funded by VicHealth and Cancer Council Victoria; the study was further supported by National Health and Medical Research Council grants 209057 and 251553 and by infrastructure provided by Cancer Council Victoria. Kelly-Anne Phillips is a National Breast Cancer Foundation Fellow.

Competing interests: No relevant disclosures. doi: 10.5694/mjal4.01203

Online first 29/09/14

1 Beral V, Bull D, Doll R, et al. Breast cancer and abortion: collaborative reanalysis of data from 53 epidemiological studies, including 83000 women with breast cancer from 16 countries. Lancet 2004; 363: 1007-1016.

2 Huang Y, Zhang X, Li W, et al. A meta-analysis of the association between induced abortion and breast cancer risk among Chinese females. Cancer Causes Control 2014; 25: 227-236.
3 Henderson KD, Sullivan-Halley J, Reynolds P, et al. Incomplete pregnancy is not associated with breast cancer risk: the California Teachers Study. Contraception 2008; 77: 391-396.

4 Michels KB, Xue F, Colditz GA, Willet WC. Induced and spontaneous abortion and incidence of breast cancer among young women: a prospective cohort study. Arch Intern Med 2007; 167: 814-820.

5 Giles GG, English DR. The Melbourne Collaborative Cohort Study. IARC Sci Publ 2002; 156: 69-70.

information

on abortions is

collected before

any of the

women develop

the disease

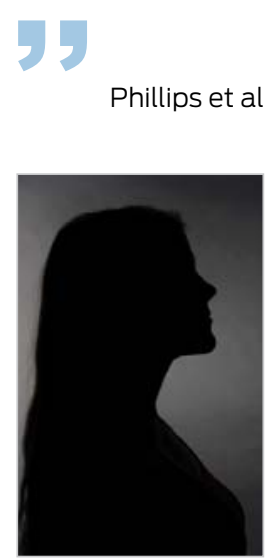

\section{New TGA warning label for use of NSAIDs in fluid-depleted children}

To THE Editor: Non-steroidal anti-inflammatory drugs (NSAIDs)

have been very widely used for many years in Australia and elsewhere, in both prescription and non-prescription settings.

Although their potential for gastrointestinal side effects is generally well understood within the community, the capacity for NSAIDs to cause renal damage, even after short-term use in susceptible individuals, is less well appreciated.

It has been well documented that the use of NSAIDs in those who are fluid-depleted, including their short-term use in otherwise healthy individuals, can lead to renal failure, albeit reversible. ${ }^{1,2}$

On 23 May this year, the Therapeutic Goods Administration updated its Medicines Advisory Statements on labels for nonprescription medicines. Included was a warning about paediatric products containing NSAIDs. ${ }^{3}$ The wording of the advisory statement is "Ask your doctor or pharmacist before use of the medicine in children suffering from dehydration through diarrhoea and/or vomiting".

As the person who initiated the request to have this warning label added, my intention was Batagol to have this warning added to all non-prescription NSAIDcontaining products for both adults and children, because we know that people who are renally compromised for any reason are 
at risk of kidney damage from the use of NSAIDs. This, of course, includes those taking some antihypertensive medications containing a diuretic, the well known "triple whammy" effect. ${ }^{4,5}$

Nevertheless, I hope that the warning label on paediatric products containing NSAIDs will alert parents and carers to be vigilant if giving these medicines to children in their care, and to check with their doctor or pharmacist if the child is fluiddepleted from diarrhoea or vomiting.

\section{Ronald P Batagol Obstetric Drug Information}

Consultant

Melbourne, VIC.

\section{healthy5555@hotmail.com}

Competing interests: No relevant disclosures. doi: 10.5694/mjal4.00959

1 John CM, Saroha V, Jones C. Using nonsteroidal anti-inflammatory drugs in volume-depleted children can precipitate acute renal failure. Pediatrics 2008; 121 Suppl 2: S142.

2 Moghal NE, Hegde S, Eastham KM. Ibuprofen and acute renal failure in a toddler. Arch Dis Child 2004; 89: 276-277.

3 Deparment of Health Therapeutic Goods Administration. Medicines Advisory Statements Specification 2014. F2014L00693. http://www.comlaw.gov.au/Details/ F2014L00693 (accessed Aug 2014).

4 Adverse Drug Reactions Advisory Committee. ACE inhibitor, diuretic and NSAID: a dangerous combination. Aust Adv Drug React Bull 2003; 22: 14-15. http://www.tga.gov.au/hp/ aadrb-0308.htm\#.U_Q8SKMUwxg (accesssed Aug 2014).

5 Kidney Health Australia. Kidney Health Australia urges caution on "triple whammy" risks [media release]. 10 Oct 2006.

\section{Will current health reforms in south and east Asia improve equity?}

To THE EDIToR: Hipgrave and Hort review health reform initiatives in south and east Asia and draw attention to the increasing privatisation of services and the inequity created as the poor have less access. ${ }^{1}$ More importantly, countries in south and east Asia are enjoying a period of economic development that has resulted in urbanisation and lifestyle change, specifically dietary change, in both urban and rural populations. This has resulted in a nutrition transition, which is associated with the chronic non-communicable disease
(NCD) "epidemic". ${ }^{2}$ The trends are that the poor in these countries will have higher levels of risk factors for chronic NCD. ${ }^{3}$

Health systems in most low and middle income countries have been designed for episodic care for acute conditions, and not for primary health care involving the continuity of care required for managing chronic disease. Second, most of these countries have made negligible investments for prevention of risk factors through population-based programs.

Third, intersectoral policies to deal with "upstream" issues, such as marketing of unhealthy food, are not yet in their reform agenda.

Australia's success in tobacco control and reduction of HIV / AIDs are lessons that we can share. However, the same cannot be said of food policy, and many countries have failed to resist the pressures of the multinational food industry. ${ }^{4}$ Countries in south and east Asia require intersectoral policies on healthy food to achieve a long-term, sustainable solution to health inequity. ${ }^{5}$

Rohan Jayasuriya Associate Professor

School of Public Health and Community Medicine, University of New South Wales, Sydney, NSW.

r.jayasuriya@unsw.edu.au

Competing interests: No relevant disclosures.

doi: $10.5694 / \mathrm{mjal} 4.00880$

1 Hipgrave DB, Hort K. Will current health reforms in south and east Asia improve equity? Med J Aust 2014; 200: 514-516.

2 Popkin BM. Global nutrition dynamics: the world is shifting rapidly toward a diet linked with noncommunicable diseases. Am J Clin Nutr 2006; 84: 289-298.

3 Popkin BM, Adair LS, Ng SW. Global nutrition transition and the pandemic of obesity in developing countries. Nutr Rev 2012; 70: 3-21.

4 Fraser B. Latin American countries crack down on junk food. Lancet 2013; 382: 385-386.

5 Popkin BM; Bellagio Meeting group. Bellagio Declaration 2013: countering Big Food's undermining of healthy food policies. Obes Rev 2013; 14 Suppl 2: 9-10.

IN REPLY: Jayasuriya draws attention to the increasing problem of non-communicable diseases (NCDs) among countries in our region, and we agree that this contributes to health inequity. Prevention of NCDs requires action across many sectors, public and private, and at state, subnational, community and individual levels. Managing NCDs creates additional challenges for effective and equitable health service delivery. However, NCDrelated services are only one example of currently ineffective primary health care in developing countries. We maintain that across the disease spectrum inadequate health reform and the lack of attention to health across sectors, the lack of public finance to provide equal access to services (including communitylevel preventive screening for and management of chronic NCDs), and inadequate attention to social determinants of health remain key to improving health equity.

\section{Countries in \\ south and east \\ Asia require \\ intersectoral \\ policies on \\ healthy food}

Jayasuriya

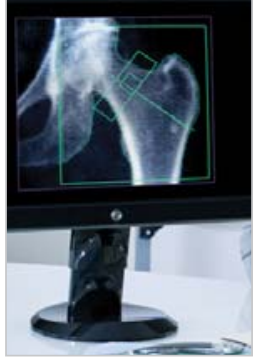

David B Hipgrave Associate

Krishna P Hort Head, Health Systems Strengthening

Unit

The Nossal Institute for Global Health, University of Melbourne, Melbourne, VIC.

dhipgrave@gmail.com

Competing interests: No relevant disclosures.

doi: 10.5694/mjal4.00996

\section{Inappropriate use of dual energy absorptiometry body composition estimation}

To THE EdToR: Bone densitometry by means of dual energy absorptiometry (DXA) has been the gold standard for estimating bone mineral density (BMD) for over 3 decades and is pivotal in the current management of osteoporosis. DXA technology relies on measuring the attenuation of two different x-ray energies that can be used to calculate BMD or, alternatively, soft tissue mass, including fat and lean tissue mass. Over the past few years, there has been increasing use of DXA for estimating fat and lean tissue in body composition. These parameters are widely used in research into diseases that affect body composition (such as HIV, obesity and eating disorders), and increasingly in related clinical settings. While using DXA in this way is beneficial in some patients, there is anecdotal evidence of growing inappropriate use or overuse of DXA body composition estimation in monitoring weight 
loss or exercise programs, often supplied by non-medical practitioners.

Two problems are worth highlighting. First, a number of body composition scan providers imply that total body BMD, provided automatically with the whole body composition scans, can provide a diagnosis of osteoporosis. This is inconsistent with World Health Organization guidelines of osteoporosis being indicated by a $\mathrm{T}$ score for bone density that is $2.5 \mathrm{SD}$ or more below the young adult mean, which applies only to the lumbar spine, proximal femur and mid shaft radius but does not apply to total body BMD. ${ }^{1}$ Applying the criterion of $\mathrm{T} \leqslant-2.5 \mathrm{SD}$ to the whole body BMD, as provided by body composition scans, will underestimate the prevalence of osteoporosis. $^{2}$

Second, some providers recommend that DXA for estimating body composition be performed 3-monthly. Although DXA for estimating body composition delivers a low radiation dose, the radiation safety principle of ALARA (As Low As Reasonably Achievable) should always be followed and there is no published evidence supporting this scan frequency. Unfortunately as DXA for estimating body composition falls outside Medicare, the federal government has no incentive to act. Moreover the low radiation dose from DXA results in relatively little attention from state radiation regulators.

Nicholas A Pocock Nuclear Medicine Physician

Weiwen Chen PhD Fellow ${ }^{2}$

1 Department of Nuclear Medicine \& PET, St Vincent's Hospital, Sydney, NSW.

2 Bone Biology, Garvan Institute, Sydney, NSW.

n.pocock@unsw.edu.au

Competing interests: Nicholas Pocock runs densitometry services which provide body composition studies.

doi: 10.5694/mjal4.00610

1 Kanis JA, Glüer CC. An update on the diagnosis and assessment of osteoporosis with densitometry. Osteoporos Int 2000; 11: 192-202.

2 Arlot ME, Sornay-Rendu E, Garnero P, et al. Apparent pre- and postmenopausal bone loss evaluated by DXA at different skeletal sites in women: the OFELY cohort. J Bone Miner Res 1997; 12: 683-690.

\section{Multiple mini interview performance of repeat applicants to medical school admission}

To THE EDITOR: As the use of the multiple mini interview (MMI) for selecting applicants for admission to health professional programs increases, so does the number of coaching classes assumed to help improve performance on the MMI. ${ }^{1}$ Moreover, applicants applying to several professional programs have multiple

opportunities to gain experience in a high-stakes environment. These realities usher concerns about the influence of "practice effect" on subsequent MMI performance. ${ }^{2}$ While short-term prior access to MMI questions did not influence applicants' performance in a Canadian medical school, ${ }^{3}$ in Australia, MMI participation in the year after the original attempt improved scores on stations (short, structured interviews to assess personal qualities such as communication, professionalism, ethics skills) that were the same or similar to original MMI stations. ${ }^{2}$

At the University of Manitoba, Canada, about $25 \%$ of 1151 unique applicants to the Faculty of Medicine's Undergraduate Medical Education program from 2008 to 2012 were those unsuccessful at gaining medical school admission in previous years. While 856 applicants (74.4\%) appeared once for the MMI, $208(18.1 \%)$ appeared twice, 64 (5.6\%) appeared thrice, $16(1.4 \%)$ appeared four times and seven $(0.6 \%)$ appeared five times. In the faculty's 12-station MMI, ${ }^{4} 88$ unique question stems or station scenarios were used in the 5-year period. The number of questions used once, twice, thrice, four and six times was 46, 18, 17, 5 and 2, respectively, with at least a 1 -year gap between original and repeat use, as recommended. ${ }^{2}$

To assess whether the scores of those who repeated the MMI were higher, applicants' average scores and within-year $z$ scores were compared by calendar year, number and order of attempts and there has been

increasing

use of DXA

for estimating

fat and lean

tissue in body

composition

Pocock et al

It is unlikely that repeat attempts ... benefited applicants

Raghavan et al then analysed by multiple linear regression. ${ }^{5}$

There was a decreasing trend in raw MMI scores for all applicants between 2008 and 2012. The mean MMI score at first attempt of one-time applicants was 4.66 (SD, 0.65 ) on a scale of 1 to 7 , while that at first attempt of 295 repeat applicants was 4.25 (SD, 0.60) $(P<0.001)$. The mean MMI score of all 707 attempts by the 295 repeat applicants improved slightly to 4.38 (SD, 0.64). After adjusting for age, sex, and calendar year, regression parameter estimates associated with 2nd, 3rd and 4th attempts, but not the 5th, gradually and significantly increased relative to the 1 st attempt. Yet, only $9.1 \%$ of the variability in within-year $z$-transformed MMI scores was explained by the selected model.

It is unlikely that repeat attempts at MMI alone systematically and considerably benefited applicants. Other unmeasured and unexplored factors, such as individuals' personal growth and maturity, may play a role in positively influencing MMI scores. Our study did not account for the effect of coaching that a substantial number of Australian medical school applicants reportedly attend., ${ }^{1,2}$ Despite some limitations in our study design and statistical methods, our results currently do not lend support to the concern that repeated MMI performance results in improved MMI scores.

Malathi Raghavan Assistant Director, ' and Assistant Professor, Community Health Sciences ${ }^{2}$

Bruce D Martin Associate Dean, Students, Undergraduate Medical Education ${ }^{2}$

1 Education and Research, American Veterinary Medical Association, Chicago, Ill, USA.

2 Faculty of Medicine, University of Manitoba, Winnipeg, Manitoba, Canada.

Malathi.Raghavan@med.umanitoba.ca

Competing interests: No relevant disclosures. doi: $10.5694 / \mathrm{mjal} 4.00728$

1 Wong CX, Roberts-Thomson RL. Does practice make perfect? The effect of coaching and retesting on selection tests used for admission to an Australian medical school [letter]. Med J Aust 2009; 190: 101-102.

2 Griffin B, Harding DW, Wilson IG, Yeomans ND. Does practice make perfect? The effect of coaching and retesting on selection tests used for admission to an Australian medical school. Med J Aust 2008; 189: 270-273.

3 Reiter HI, Salvatori P, Rosenfeld J, et al. The effect of defined violations of test security on admissions outcomes using multiple miniinterviews. Med Educ 2006; 40: 36-42. 
4 Raghavan M, Burnett M, Martin BD, et al. Utility of a writing station in the multiple miniinterview. J Vet Med Educ 2013; 40: 177-183.

5 Bewick V, Cheek L, Ball J. Statistics review 7: Correlation and regression. Crit Care 2003; 7 : 451-459.

\section{Time to end the ban on HIV-positive proceduralists and dentists}

To THE EdToR: This year, the ban on HIV-positive surgeons and dentists practising in the United Kingdom was removed on the provision that they are clinically well, are being treated, and have an undetectable viral load. ${ }^{1}$ This development aligns the UK with over 20 other countries. Australia is lagging behind other more progressive countries on this issue. $^{2}$

During the height of the HIV epidemic in the early 1990s, there were no HIV transmissions among 22171 patients exposed to preantiretroviral therapy (ART)-era HIV-positive doctors and dentists during invasive operations. ${ }^{3}$

There have been only 10 published case reports indicating probable transmission from a proceduralist to patients since HIV was first reported. ${ }^{4}$ The current risk estimate is 1 in $1672000 .^{2} \mathrm{No}$ cases of inadvertent transmission have been reported in the literature from countries that allow HIV-positive proceduralists. ${ }^{2}$

ART is vital for reducing the risk of transmission. Extrapolating from the ongoing PARTNER study, there have been no transmissions between serodiscordant couples who have regular, unprotected, high-risk sex if the positive partner is receiving ART and virally suppressed 2 years into the study. ${ }^{5}$ The risk during procedures by HIV-positive virally suppressed proceduralists, with the use of standard universal precautions (such as gloves and sterile equipment), would likely be extremely low.

There are two major problems with a blanket ban on HIV-positive proceduralists. First, those facing a career-ending outcome of a test are likely to avoid being tested after an initial negative test result. Second, the wrong message is sent to the public that people with HIV are highly infectious and are somehow dangerous (despite treatment). Lifting the ban would lead to greater incentives for proceduralists to be regularly tested for HIV, leading to better outcomes for them and their patients. This is important as many infected individuals transmit the virus when they are seroconverting and are unaware of their diagnosis. ${ }^{6}$ Furthermore, postexposure prophylaxis could also be available to patients after possible exposure during a procedure by following occupational exposure guidelines.

It is time for Australia to align with other progressive nations and end the absolute ban on HIV-positive proceduralists. The risk of transmission from them, when treated for HIV and using standard precautions, is likely to be negligible.

Sean R Atkinson Sexual Health Registrar Department of Sexual Health, Cairns Hospital, Cairns, QLD. drseanatkinson@gmail.com

Competing interests: No relevant disclosures. doi: 10.5694/mjal4.01031

1 Ban lifted on HIV-positive doctors practising surgery. (news). London: British Medical Association, 2013. http://bma.org.uk/newsviews-analysis/news/2013/august/ban-liftedon-hiv-positive-doctors-practising-surgery (accessed Sep 2014).

2 Ma R. Discrimination against doctors with HIV must end. BMJ 2012; 344: e3440.

3 Robert LM, Chamberland ME, Cleveland JL, et al. Investigations of patients of healthcare workers infected with HIV: The Centers for Disease Control and Prevention database. Ann Intern Med 1995; 122: 653-657.

4 Centers for Disease Control and Prevention. Investigation of patients treated by an HIVinfected cardiothoracic surgeon - Israel, 2007. MMWR Morb Mortal Wkly Rep 2009; 57 1413-1415.

5 Rodger A, Bruun T, Cambiano V, et al. HIV transmission risk through condomless sex if HIV+ partner on suppressive ART: PARTNER study. 21st Conference on Retroviruses and Opportunistic Infections; 2014 Mar 3-6; Boston, Mass, US; abstract 153LB.

6 Chibo D, Kaye M, Birch C. HIV transmissions during seroconversion contribute significantly to new infections in men who have sex with men in Australia. AIDS Res Hum Retroviruses 2012; 28: 460-464.

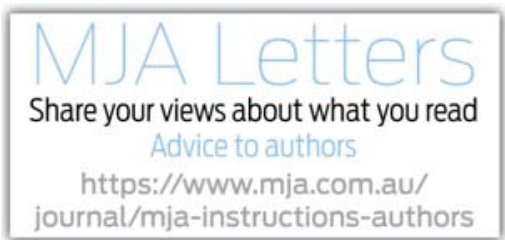

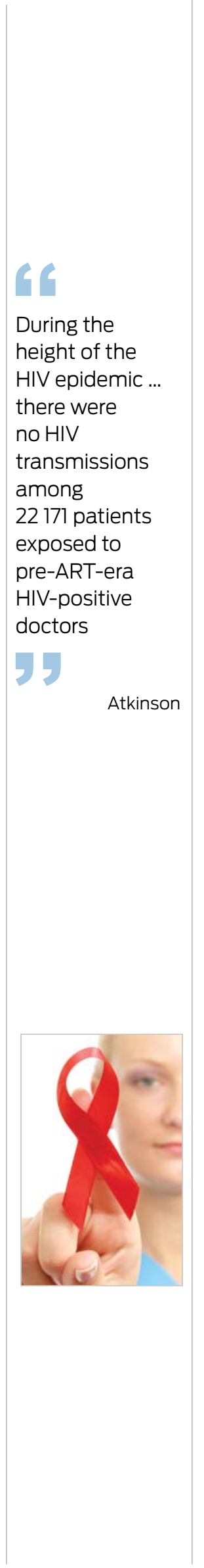

